\title{
Toward Quantification and Visualization of Active Stress Waves for Myocardial Biomechanical Function Assessment
}

\author{
Niels F Otani ${ }^{1}$, Dylan Dang ${ }^{1}$, Christopher Beam ${ }^{1}$, Fariba Mohammadi ${ }^{2}$, Brian Wentz ${ }^{3}$, S M Kamrul \\ Hasan $^{4}$, Suzanne M Shontz ${ }^{5}$, Karl Q Schwarz ${ }^{6}$, Sabu Thomas ${ }^{6}$ and Cristian A Linte ${ }^{* 4,7}$ \\ ${ }^{1}$ School of Mathematical Sciences, Rochester Institute of Technology, Rochester NY; ${ }^{2}$ Mechanical \\ Engineering, University of Kansas, Lawrence, KS; ${ }^{3}$ Bioengineering Graduate Program, University of \\ Kansas, Lawrence, KS; ${ }^{4}$ Center for Imaging Science, Rochester Institute of Technology, Rochester, \\ $\mathrm{NY} ;{ }^{5}$ Electrical Engineering and Computer Science, University of Kansas, Lawrence, KS; ${ }^{6}$ Division \\ of Cardiology, University of Rochester Medical Center, Rochester, NY; ${ }^{7}$ Biomedical Engineering, \\ Rochester Institute of Technology, Rochester, NY, USA
}

\begin{abstract}
Estimating and visualizing myocardial active stress wave patterns is crucial to understanding the mechanical activity of the heart and provides a potential non-invasive method to assess myocardial function. These patterns can be reconstructed by analyzing $2 D$ and/or $3 D$ tissue displacement data acquired using medical imaging. Here we describe an application that utilizes a $3 D$ finite element formulation to reconstruct active stress from displacement data. As a proof of concept, a simple cubic mesh was used to represent a myocardial tissue "sample" consisting of a $10 \times 10 \times 10$ lattice of nodes featuring different fiber directions that rotate with depth, mimicking cardiac transverse isotropy. In the forward model, tissue deformation was generated using a test wave with active stresses that mimic the myocardial contractile forces. The generated deformation field was used as input to an inverse model designed to reconstruct the original active stress distribution. We numerically simulated malfunctioning tissue regions (experiencing limited contractility and hence active stress) within the healthy tissue. We also assessed model sensitivity by adding noise to the deformation field generated using the forward model. The difference image between the original and reconstructed active stress distribution suggests that the model accurately estimates active stress from tissue deformation data with a high signal-to-noise ratio.
\end{abstract}

\section{Introduction}

Under normal conditions, the heart contracts in response to electrical waves that propagate through the cardiac muscle, elicit calcium ion release in response to the membrane depolarization, and cause the contraction of the cardiomy- ocytes, which, in turn, give rise to the cardiac tissue deformations. A wide variety of cardiac conditions manifest themselves by a lower than normal myocardial contractility, which, in the long run, leads to heart failure. Such conditions are typically associated with tissue regions whose viability has been compromised by either inadequate blood perfusion (i.e., blockage of one or more of the coronary vessels that supply blood to the myocardium) or malfunction at the electro-physiological level, leading to abnormal contraction patterns.

Although often used as a surrogate for function, cardiac electrophysiology is at least "once removed" from the direct biomechanical response of the tissue. Not all cardiac conditions that result in reduced cardiac function originate at the electrophysiology level. Sub-optimal contractions may occur in response to reduced biomechanical tissue viability (i.e., the ability to contract normally), rather than in response to abnormal electrical activity. Such conditions are characterized by poorly contracting myocardial regions, despite their typically normal electrophysiology.

Ischemic regions, which exhibit diminished contractile function, are detected using one of several methods: 1) delayed contrast-enhanced MR imaging [1]; 2) strain imaging [2-4]; or 3) nuclear metabolic imaging [5,6]. Delayed contrast-enhanced MR imaging renders non-viable tissue regions as hyper-enhanced areas due to the poor washout of the contrast agent pooling in from the extracellular space [7]. Ischemic regions are identified and classified according to their size and transmural extent, but no information is available on the biomechanical viability of the tissue (i.e., its ability to generate normal active stresses). Strain imaging relies on the processing of either ultrasound or MR images to estimate deformation during the cardiac cycle. While this method may point out hypo-kinetic or dyskinetic segments, their motion is 
compromised by the passive motion of the surrounding, functional tissue. Lastly, functional assessment by quantifying the uptake of an injected radioactive tracer using nuclear imaging (i.e., positron emission tomography) techniques helps identify regions experiencing lower than normal metabolic activity, which may be indicative of reduced viability. While these techniques have shown some correlation to tissue hypo-perfusion, which, in turn, lead to speculations regarding tissue viability, none of the above techniques provide direct estimates of tissue viability (i.e., measure of actual force of contraction), but rather surrogates based on tissue perfusion, tissue motion or tissue metabolic activity. Thus, there is a need for a method that directly quantifies the cardiac biomechanical function at a larger scale than electrophysiology. Such a method would enable direct assessment of the biomechanical activity or work done by the heart, based on the cardiac deformations and biomechanical tissue properties.

We hypothesize that estimates of myocardial active stresses reconstructed using computational biomechanics, given the myocardial deformation field (i.e., extracted using medical imaging) [8] and mechanical and physiological properties of cardiac tissue (i.e., tissue elasticity and fiber isotropy), will better help reveal the underlying contractile mechanism of the myocardium and assess tissue viability based on a direct, quantitative measure (Fig. 1) [9]. Therefore, we propose a technique to estimate the active stresses developed within the myofibers as a means to provide a direct measure of the work output - a direct quantitative measure of tissue viability [10].

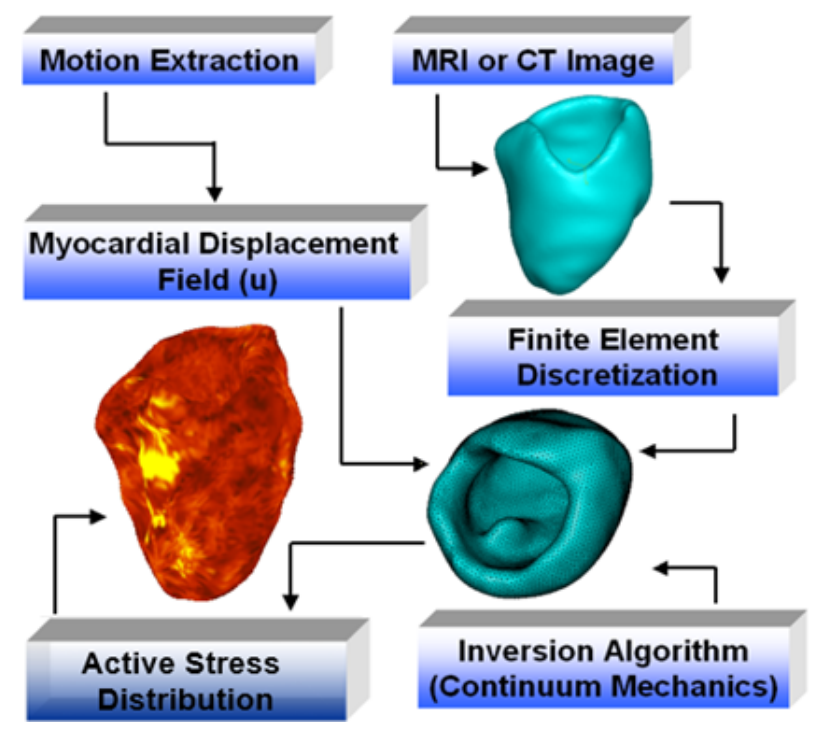

Figure 1. Schematic representation of the proposed method for active stress reconstruction given the 4D myocardial deformation field (i.e., strain).

\section{Methods}

Within the larger scope of this work focused on reconstructing, quantifying and visualizing myocardial active stresses by solving an inverse biomechanics problem subject to input cardiac deformations extracted using medical imaging, this paper resumes to the initial implementation and validation of the 3D finite element formulation to reconstruct the active stresses from displacement data in response to action potential wave propagation in a simple cubic mesh (Fig. 2) used to represent a "sample" of myocardial tissue consisting of a $10 \times 10 \times 10$ lattice of nodes featuring different fiber directions that rotate with depth, mimicking transverse isotropy.

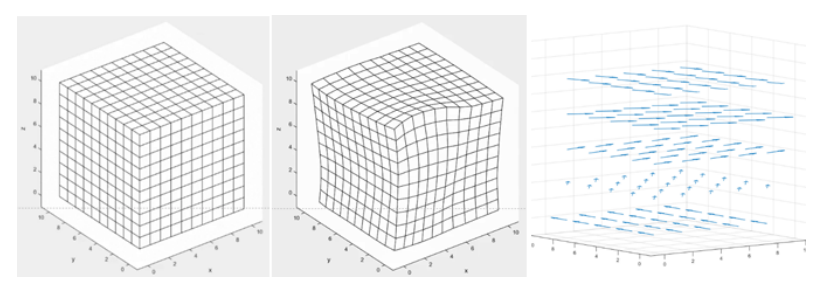

Figure 2. Representation of the synthetic cardiac tissue model consisting of a $10 \times 10 \times 10$ lattice of nodes featuring different fiber directions that rotate with depth (i.e., in this case the $z$-axis), mimicking transverse isotropy.

In the forward model, tissue deformation was generated using a test wave with active stresses that mimic the myocardial contractile forces. Two of the three components of the deformation field were used as input to an inverse model designed to reproduce the original active stress distribution.

The cardiac biomechanical model we propose is based on fundamental relationships among the tissue strain (including the deformation and contraction of the heart), passive stress response to the strain, and the active stress produced by the electrical (action potential) wave. This relationship is expressed by two equations:

$$
\frac{\partial}{\partial X_{M}}\left[\left(T_{M N}(E)+T_{M N}^{a c t i v e}-p C_{M N}^{-1}\right) \frac{\partial x_{j}}{\partial X_{N}}\right]=0,
$$

which expresses force balance among the active and passive stresses and the hydrostatic pressure, and

$$
\operatorname{det}(F)=1,
$$

which, together with the hydrostatic pressure in Eq. (1), enforces incompressibility [11].

Here $X_{N}$ and $x_{j}(N, j=1,2,3)$, are the coordinates in the un-deformed and deformed coordinate systems, respectively, $T_{M N}(E)$ is the passive second Piola-Kirchhoff stress tensor, which depends on the strain tensor $E, p$ is the local hydrostatic pressure, $F_{j M} \equiv \partial x_{j} / \partial X_{M}$ is the deformation gradient tensor, $C=F^{T} F$, and $T_{M N}^{a c t i v e}$ is the 
stress tensor due to the action potential induced force generated parallel to the orientation of the myocardial fibers.

We neglect inertia in Eq (1) because we are interested only in low-frequency motion and not mechanical waves, and also assume incompressibility, as we currently have no reason to believe that the slightly reduced volume of the tissue due to expulsion of blood from the cardiac muscle during the cardiac cycle plays a substantial role. For the passive-stress-strain relationship, $T_{M N}(E)$, we use the hyperelastic Nash-Hunter cardiac model [12].

Equations (1) and (2) form the basis for solving both the forward problem, in which the active stresses are known as functions of time and space, and the displacements (i.e., the tissue deformations) and hydrostatic pressures produced by the active stresses are calculated. These equations can be used to solve the inverse problem, wherein the active stresses and the hydrostatic pressures are calculated given the deformations. Solving these equations is equivalent to applying the principle of virtual work to an energy integral. Hence we require that the net energy be zero with respect to virtual displacements in $x_{j}$, subject to an incompressibility constraint.

In general, the calculation of active stresses from displacements using Eqs. (1) and (2) is usually an underdetermined problem in more than one spatial dimension. However, for the heart, the problem is overdetermined, as shown by Otani et al. in [11], since the active stress is directed solely along the local fiber direction. Therefore, since only one component of the active stress tensor in the local fiber-direction frame is non-zero, the problem is then overdetermined, and may be solved as a least-squares problem using the technique described in $[11,13]$. Moreover, the substantial reduction in the number of unknowns not only allows us to find a unique solution, but also affords improved confidence in the solution in the presence of noise.

\section{Results}

We have successfully implemented finite element, linearized versions of Eqs. (1) and (2) as both forward and inverse problems [11] using this technique in rectangular geometry with stress-free boundary conditions with fiber directions that rotate in the $x-y$ plane as functions of the depth $z$ (Fig. 3). Using this implementation, in the forward model, tissue deformation was generated using a test wave with active stresses that mimic the myocardial contractile forces. Two of the three components of the deformation field were used as input to an inverse model designed to reproduce the original active stress distribution.

We conducted several simulations to numerically validate our inverse model. First, we tested the algorithm when no noise was present. Fig. 4 shows the active stress wave reconstructed using our algorithm using the displacements

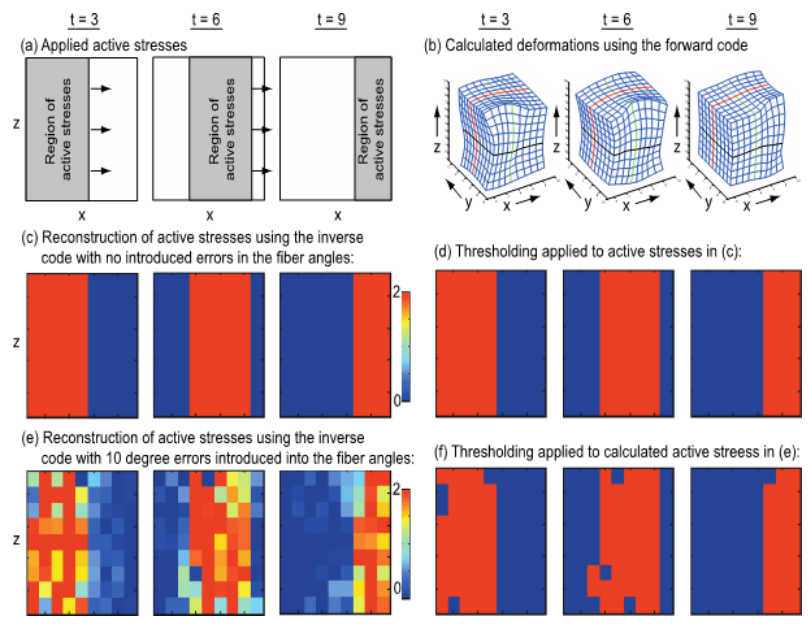

Figure 3. Representation of the synthetic cardiac tissue model consisting of a $10 \times 10 \times 10$ lattice of nodes featuring different fiber directions that rotate with depth (i.e., in this case the $z$-axis), mimicking transverse isotropy.

generated by the forward model as input, which is identical to the the active stress wave prescribed as input into the forward model.

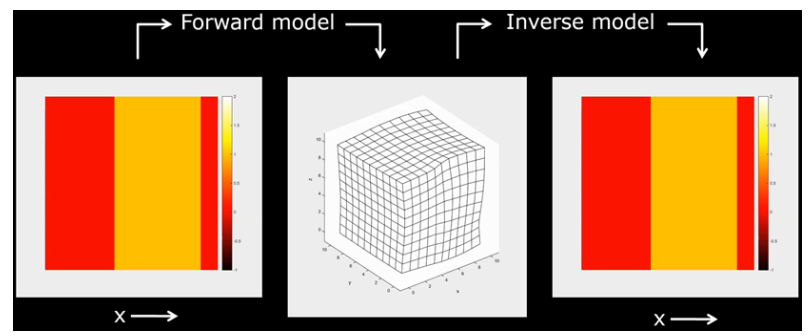

Figure 4. Forward model and inverse reconstruction of active stress action potential wave in presence of no noise at $10 \times 10 \times 10$ lattice node resolution.

To assess model sensitivity to noise, we applied Gaussian noise with a $1 \%$ standard deviation to the displacement fields generated using the forward model, then we used the inverse model to reconstruct the active stress wave. As shown in Fig. 5, the reconstructed active stress distribution features a high frequency checkerboard error pattern, but the overall wave behavior is not compromised.

Lastly, since this method is intended to identify subcontractile myocardial regions, we numerically simulated tissue regions that do not generate active stress. We then used the displacement fields generated using the forward model as input into the inverse model to reconstruct the active stress waves. Fig. 6 shows the "abnormal" tissue regions experiencing low active stress were successfully reconstructed despite the checkerboard pattern noise. Moreover, a two-fold resolution increase significantly reduced noise amplitudes, rendering a more clear identification of 


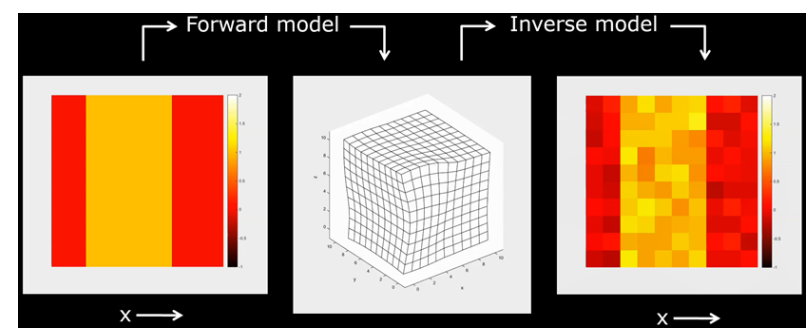

Figure 5. Forward model and inverse reconstruction of active stress action potential wave in presence of $1 \%$ standard deviation noise in the node displacement field.

the sub-contractile regions.

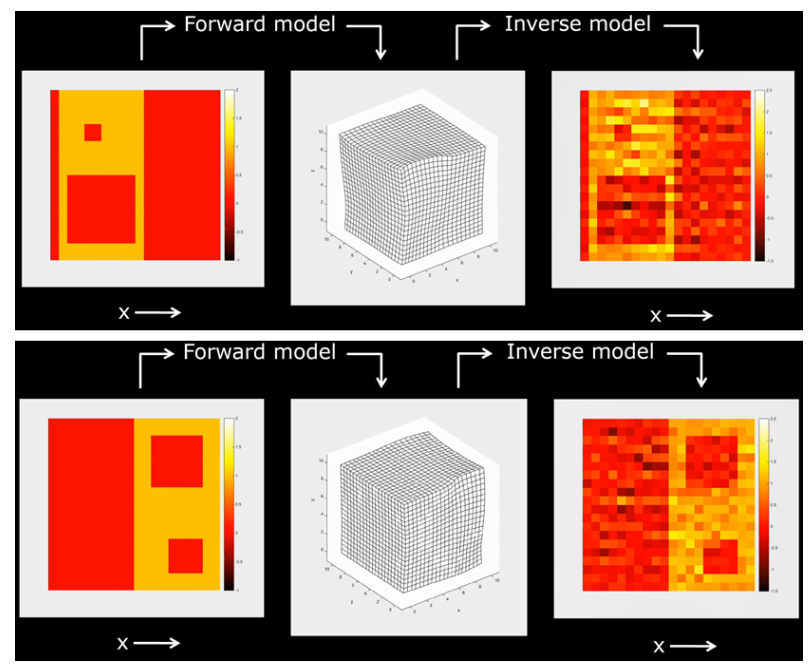

Figure 6. Forward model and inverse reconstruction of active stress action potential wave in presence of subcontractile regions at $20 \times 20 \times 20$ node resolution.

\section{Conclusions and Future Work}

The model accurately estimates active stresses from tissue deformation data with a high signal-to-noise ratio. The error pattern is expected to follow the Nyquist criterion, suggesting that model would provide reasonable results at depicting details that are at least two computation units in size. Future work entails model implementation using a more realistic geometry obtained by segmenting the left ventricle myocardium from clinical quality $3 \mathrm{D}$ and $4 \mathrm{D}$ medical images, as well as further reducing noise in the inverse reconstruction. Furthermore, the algorithm will be implemented using a high-order, curvilinear left ventricle mesh that faithfully preserves the geometry and shape of the anatomy, and also includes fiber architecture.

\section{Acknowledgements}

This work was supported by grants from the National Science Foundation (Award No. 1808530 \& 1808553) and the National Institutes of Health (Award No. R35GM128877).

\section{References}

[1] Hillenbrand HB, Kim RJ, Parker MA, Fieno DS, Judd RM. Early assessment of myocardial salvage by contrastenhanced magnetic resonance imaging. Circulation 2000; 102:1678-83.

[2] Voigt JU, Arnold MF, Karlsson M, Hubbert L, Kukulski T, Hatle L, Sutherland GR. Assessment of regional longitudinal myocardial strain rate derived from doppler myocardial imaging indexes in normal and infarcted myocardium. J Am Soc Echocardiogr 2000;13:588-98.

[3] Konofagou EE, Dhooge J, Ophir J. Myocardial elastography - a feasibility study in vivo. Ultrasound Med Biol 2002; 28:475-82.

[4] McVeigh ER. Mri of myocardial function: motion tracking techniques. Magn Reson Imaging 1996;14:137-50.

[5] Matsunari I, Taki J, Nakajima K, Tonami N, Hisada K. Myocardial viability assessment using nuclear imaging. Ann Nucl Med 2003;17:169-79.

[6] Pirich C, Schwaiger M. The clinical role of positron emission tomography in management of the cardiac patient. Rev Port Cardiol 2000;19(Suppl. 1):I89-100.

[7] Mahnken AH, Koos R, Katoh M, Wildberger JE, Buecker A, Gunther RW, Kuhl HP. Assessment of myocardial viability in reperfused acute myocardial infarction using 16slice computed tomography in comparison to magnetic resonance imaging. J Am Coll Cardiol 2005;45:2042-7.

[8] Provost J, Lee WN, Fujikura K, Konofagou EE. Electromechanical wave imaging of normal and ischemic hearts in vivo. IEEE Trans Med Imaging 2010;29:625-35.

[9] Linte CA, Wierzbicki M, Peters TM, Samani A. Towards a biomechanics-based technique for assessing myocardial contractility: an inverse problem approach. Comput Methods Biomech Biomed Eng 2008;11:245-55.

[10] Otani NF, Dang D, Dangil S, Stees M, Shontz SM, Linte CA. Assessing cardiac tissue function via action potential wave imaging using cardiac displacement data. Lect Notes Comput Vision Biomech 2017;27:903-12.

[11] Otani NF, Luther S, Singh R, Gilmour Jr. RF. Transmural ultrasound-based visualization of patterns of action potential wave propagation in cardiac tissue. Ann Biomed Eng 2010;38:3112-23.

[12] Nash MP, Hunter PJ. Computational mechanics of the heart. J Elast 2000;61:113-41.

[13] Muñoz LM, Stockton J. F. an Otani NF. Applications of control theory to the dynamics and propagation of cardiac action potentials. Ann Biomed Eng 2010;38:2865-76.

Address for correspondence:

${ }^{*}$ Cristian A. Linte, PhD. RIT - Institute Hall (73) Rm 3111, Rochester NY 14467 USA. Email: clinte@mail.rit.edu. 